\title{
Gansang Stone Inscriptions: A New Discovery That May Change the History of the Tai-Kadai Ethnic Groups
}

\author{
Jianghua $\operatorname{Han}^{1}$ \\ ${ }^{1}$ College of Literature and Journalism, Sichuan University, Chengdu, China \\ Correspondence: Jianghua Han, College of Literature and Journalism, Sichuan University, No.24 South Section \\ 1, Yihuan Road, Chengdu City, Sichuan Province, China. E-mail: hanjianghua@scu.edu.cn
}

Received: June 17, 2019

Accepted: July 3, 2019 Online Published: July 23, 2019

doi:10.5539/ass.v15n8p45

URL: https://doi.org/10.5539/ass.v15n8p45

This work was supported by the Sichuan University, under Grant number: 2018hhs-38.

\begin{abstract}
The discovery of the Gansang stone inscriptions is the most important ancient character discovery in China since that of the oracle-bone inscriptions. It has had a major impact on research on ancient characters in China, and it will also have serious consequences for the study of human civilization. The discovery makes it possible to rewrite the history of the ancient Tai-Kadai ethnic groups in Southwest China, which were previously thought to have no direct written history. Radiocarbon dating of the stone tablets indicates that the Gansang stone inscriptions have a history of about 3,000 years. Scholars agree that the Gansang stone inscriptions display an ancient ideographic writing system of the ancient Tai-Kadai ethnic groups and that they date to almost the same era as the oracle-bone inscriptions. While the position of stroke movements in the inscriptions has been determined, it is unclear whether the texts are arranged from left to right or right to left. A comparative analysis of the Gansang stone inscriptions, the oracle-bone inscriptions, the Shuishu writing system, and the ancient Yi writing system indicates that the Gansang stone inscriptions recorded people's apparel, architecture, residence, eating habits, transportation, hunting activities, war, raising livestock, sacrifice, divination, astronomy, and calendar.
\end{abstract}

Keywords: Gansang Stone Inscriptions, Tai-Kadai Ethnic Groups, Civilization

\section{Gansang Stone Inscriptions: An Accidental Discovery Opens the Door to a New Civilization}

According to the Pingguo County Government of Guangxi, China, the Gansang stone inscriptions were accidentally discovered by the villager Rongguan Pan who lived in Gansang Village, Matou Town, Pingguo County. From October 2006 to October 2011, Pan discovered and collected more than 20 pieces of stone tablets engraved with various symbols. Pan, who has junior high school-level literacy, did not know what the symbols were, but believed that they must have some specific meaning. Thus, he sent the stone tablets to the relevant departments of Pingguo County Government and Baise City Government for identification.

Pan's requests did not immediately attract the attention of the relevant departments. Later, Haihua Feng, a member of the Guangxi Luoyue Culture Research Association, carefully studied the symbols on the stone tablets and thought that they might be the characters used by the ancient Luoyue people (a branch of the ancient Tai-Kadai ethnic groups). This preliminary appraisal attracted the attention of the Guangxi Luoyue Culture Research Association. On December 19, 2011, experts were organised to investigate the stone inscriptions, and a large number of stone tablets engraved with various symbols were collected as research materials.

Later, in February 2012, with the strong support of the Pingguo County Government, a team of archaeological researchers from The Institute of Cultural Relics and Archaeology of Guangxi Autonomous Region, the Ethnic Museum of Youjiang City, and the Museum of Pingguo County conducted archaeological excavations at the stone carving sites. After more than four months of excavation work, many pieces of stone engraved with symbols were discovered.

Experts worked together to systematically list and copy these carved symbols and named them the Gansang stone inscriptions based on the name of the place in which they were discovered. Zhiqiang Li (2018) included 16,173 characters from the Gansang stone inscriptions in his book "The image narrative of Pingguo Gansang stone inscriptions: Characters copy set." Li (2018) pointed out that some stone inscriptions were forced to be 
abandoned due to their lack of clarity, while others could not be collected or copied, as they are in the hands of private collectors (p. 5). Counting these inscriptions that are missing from Li's book, the characters of Gansang stone inscriptions should add up to about 18,000 characters (Li, 2018, p. 5).

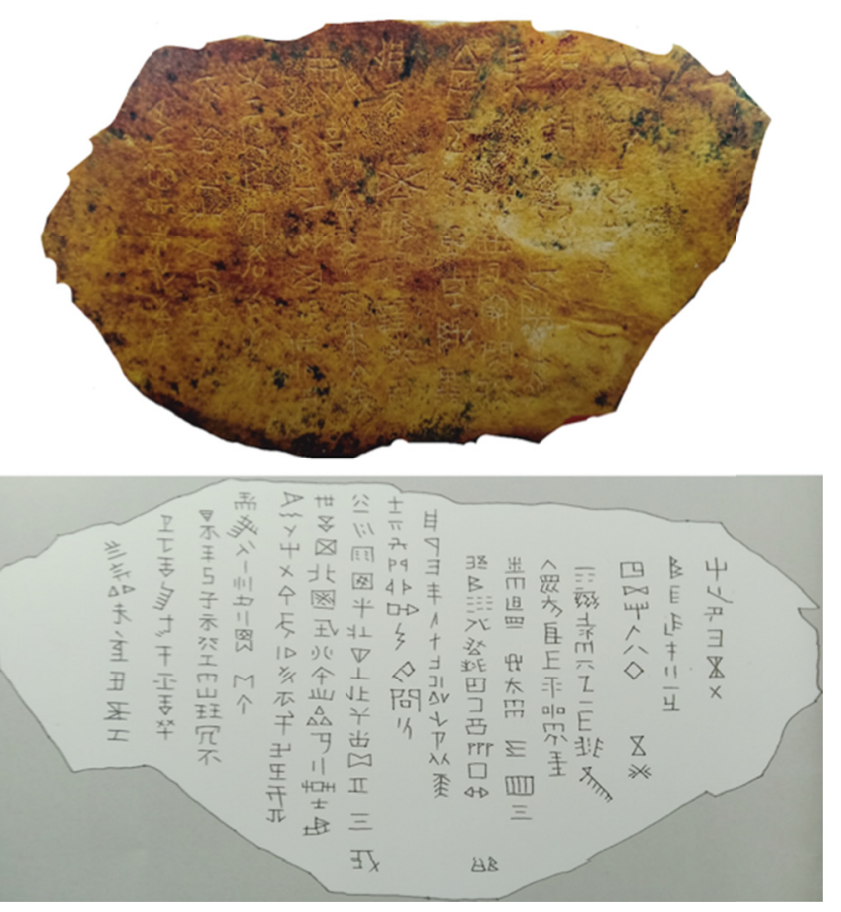

Figure 1. Sample of Gansang stone inscriptions and manual copy (Ban, 2013, p. 4, 24)

Many scholars, such as Qingxia Dai (2013), Cao Ban (2013), Jinfang Li and Ye Liu (2014), Tingwang Liang (2013, 2018), and Zhiqiang Li $(2016,2018)$ believe that the discovery of Gansang stone inscriptions is one of the most important discoveries of ancient characters in China since the discovery of the oracle-bone inscriptions; it will have a major impact on our understanding of the development of ancient Chinese characters and that of the history of human civilization. With this discovery, it is possible to rewrite the history of the ancient Tai-Kadai ethnic groups in Southwest China, which had been thought to have no direct written history until now. The Gansang stone inscriptions opened a new door for the exploration of civilization.

\section{The Nature and Historical Status of the Gansang Stone Inscriptions}

After the Gansang stone inscriptions were discovered, scholars studied them from various perspectives in order to determine their nature and historical status and lay the foundation for the correct interpretation of the Gansang stone inscriptions in the future.

\subsection{An Investigation into the Age of the Gansang Stone Inscriptions}

After the Gansang stone inscriptions were discovered, scholars began to attempt to determine their age. Since it is still impossible to interpret the inscriptions and no relevant historical documents exist, the age of the Gansang stone inscriptions could not be determined using methods from the humanities. Thus, scholars turned to the natural sciences and hoped to determine the age of the inscriptions by testing the radiocarbon (14C) of the stone tablets. Using radiocarbon data, it was determined that the fifth layer (calcification) of the stone tablets is aged $2,950 \pm 167$ years and that the fourth layer (shell) of the stone tablets is aged 3,680 \pm 172 years (Liang, 2013).

Of course, these are only the results of preliminary tests by scientific institutions. While the results indicate that these stone tablets date to the specified era, this does not mean that the Gansang stone inscriptions definitely appeared in that era. The age of the Gansang stone inscriptions may be earlier or later than the age of the formation of the stone tablets. Therefore, the assumed age of the Gansang stone inscriptions may be relatively inaccurate until scholars correctly interpret them.

\subsection{Zhuang Ethnic Folk Ancient Scripts and Gansang Stone Inscriptions Research Symposium: Preliminary Research Results of the Gansang Stone Inscriptions}

The study of the Gansang stone inscriptions is still in the initial stage, and the research foundations are very weak. Papers that presented in-depth academic research on the Gansang stone inscriptions first appeared at the 
academic conference "Zhuang ethnic folk ancient scripts and Gansang stone inscriptions research symposium," which was held in Pingguo County on January 25-27, 2013.

In this conference, Tingwang Liang (2013) pointed out in the report "Gansang cared symbols: The characters of ancient Luoyue are immortal" that the place in which the Gansang stone inscriptions were discovered is the same location in which the ancient Luoyue ethnic group, the ancestors of the Zhuang ethnic group, lived for generations. The geographical location is remote and the surrounding mountains isolate it from the outside world; there is no historical record of immigration from outside. Considering these factors, Tingwang Liang indicated that the Gansang stone inscriptions may be the writing system of the ancient Luoyue ethnic group. In addition, Zhongqun Qin (2013) pointed out in "The report of the identification of Gansang stone inscriptions by Yi (Lolo) expert" that some Yi (Lolo) experts believe that the Gansang stone inscriptions have a close relationship with the ancient Yi (Lolo) writing system, as some characters from the Gansang stone inscriptions can be matched with those from the ancient Yi (Lolo) writing system. However, grammar rules have not been determined and the inscriptions in each stone tablet cannot be completely read, so it is impossible to draw conclusions about the meaning and attribution of the Gansang stone inscriptions.

According to historical records, Pingguo County has historically been the home of the ancient Luoyue ethnic group, and there is no historical record of immigration from outside (He, 2006). However, we are still unable to confirm the status of communication between the Luoyue ethnic group and the outside world. While the current literature shows that Pingguo County had no outside immigration in ancient times, this does not mean that the ancient Luoyue ethnic group living there had no contact with the outside world. Therefore, considering the assumption of no historical record of immigration from outside, the argument that the Gansang stone inscriptions display the Luoyue (ancient Tai-Kadai) writing system lacks persuasiveness.

Further comparative studies are required to confirm that the Gansang stone inscriptions have a close relationship with the ancient Yi (Lolo) writing system. At present, we cannot rule out the possibility of accidental similarities between the ancient Yi (Lolo) writing system and the Gansang stone inscriptions. In addition, academic circles are still uncertain of the age of the ancient Yi (Lolo) writing system. Therefore, to clarify the relationship between the Gansang stone inscriptions and the ancient Yi (Lolo) writing system, it may be necessary for scholars of the ancient Yi (Lolo) writing system to cooperate with scholars studying the Gansang stone inscriptions. This would promote the deepening of research on both the ancient Yi (Lolo) writing system and the Gansang stone inscriptions.

2.3 The Research Team of Gansang Ancient Civilization and Gansang Stone Inscriptions in Baise College: The First Formal Professional Research Team Studying the Gansang Stone Inscriptions

In April 2013, Baise College established the Gansang ancient civilization and Gansang stone inscriptions research team. Members of the research team came from many related universities and research institutes across China. After its establishment, the research team actively organised researchers to carry out extensive and in-depth field investigations collecting and sorting archaeological materials, physical objects, carved stone tablets, and literature materials related to the Gansang stone inscriptions. After years of accumulation and research, the research team has made many new discoveries:

(1) The research team conducted a survey on anthropology, folklore, and sociology from July 12 to 16, 2013 in Gansang village, Banke village, and Sanban village of Pingguo county. During the investigation, the team members collected a large number of stone shovels, stone adze, and stone tablets engraved with various characters. After the investigation, the research team sent these stones to Fengxian Huang, Vice President of the Chinese Ancient Characters Society, for identification. Huang then confirmed that these are genuine Gansang stone inscriptions (Li, 2018, p. 5). Later, Chaoxiong Zheng (2014), the Guangxi Museum Director, further studied the stone inscriptions collected by the research team and pointed out that they are similar to the bronze inscriptions of the Western Zhou Dynasty in ancient China. The discovery of these stone inscriptions laid a solid foundation for the correct interpretation of the Gansang stone inscriptions.

(2) In the field survey of 2013, members of the research team collected an ancient legend through an interview with Xianqun Huang, the party secretary of Sanban village. The legend that states that the Sambo created characters (a writing system) to teach people Liao songs.

Liao songs are a type of ancient folk from the Zhuang ethnic group. They originated in Pingguo county and can be traced back to the Tang and Song Dynasties. Liao songs mainly reflect the production, life, love, marriage, history, and other aspects of the Zhuang people (Zheng, 2005). As part of China's national intangible cultural heritage, Liao songs are an important part of Zhuang culture and inherit the cultural code of the Zhuang ethnic group. Liao songs are recorded in ancient Zhuang characters, and scholars have found that hundreds of radical 
ancient Zhuang characters are not related to Chinese characters. After comparing the texts of these Liao songs with the Gansang stone inscriptions, the research team believes that the radicals of the non-Chinese original characters in the ancient Zhuang characters may match those in the Gansang stone inscriptions. Of course, this view is only a speculation at present, and identifying the correct conclusion will require further research and argumentation.

(3) The members of the research team also collected and sorted folk myths and legends that were recorded in ancient Zhuang characters in the 2013 field survey. The most representative among these ancient Zhuang character documents is the "Epic Buluotuo." Each section of the "Epic Buluotuo" starts with the phrase, "Three realms are made by three kings, four realms are made by four kings" (Huang, 2016, p. 3). The "three kings" here are the thunder tribes, the bird tribes, and the scaly dragon tribes. The Buluotuo is the leader of the bird tribes, and in the early Shang Dynasty, the bird tribe alliance group built Luoyue joint city-state country (Li, 2018, p. 5). According to these documents, Zhiqiang Li (2018) pointed out that the Gansang stone inscriptions may be the source of the "Epic Buluotuo" and the other scriptures written in ancient Zhuang characters (p. 6). Of course, this view is only based on speculation considering limited materials and merely provides a direction and perspective for the study of the Gansang stone inscriptions. Due to the lack of relevant evidence, it is still impossible to make a rigorous scientific argument for this perspective.

2.4 The Contention of a Hundred Schools of Thought: An Overview of Scholars' Interpretations of the Gansang Stone Inscriptions

\subsubsection{Views of Tingyu Jiang (Former Director of the Guangxi Museum)}

After studying the Gansang stone inscriptions, Tingyu Jiang (2013) asserted that the Gansang stone inscriptions were the characters of the pre-Qin dynasty of China. He made this judgment because there are many similarities between the Gansang stone inscriptions and the oracle-bone inscriptions in the Yin Shang dynasty of China, and because the Gansang stone inscriptions do not appear to have the same characteristics as the Seal Script and Clerical Script of Chinese characters. This analysis is consistent with the test results of the radiocarbon dating of the stone tablets engraved with the Gansang stone inscriptions. However, Jiang's analysis also lacks fully convincing evidence; we cannot prove that the writing system of ethnic groups that appeared after the Qin Dynasty must have the characteristics of the Seal Script and Clerical Script of Chinese characters.

\subsubsection{Views of Fang Qin (Researcher at the Guangxi Institute of Cultural Relics and Archaeology)}

Fang Qin (2013) compared the Gansang stone inscriptions with images in the Zuojiang murals, the inscriptions on the bronze wares of the Warring States Period unearthed in the Lingnan area of China, and the inscriptions on pottery unearthed in the Guangxi area of China. Qin found that there are five images in the Gansang stone inscriptions similar to the Zuojiang murals and found 17 symbols similar to the word “王 (King)" carved on the bronze wares of the Warring States Period unearthed in the Lingnan area. Qin also found many similarities between the Gansang stone inscriptions and the inscriptions on pottery unearthed in the Guangxi area. According to this research, Fang Qin asserted that the Gansang stone inscriptions are part of the writing system created by the ancient Luoyue ethnic group during the Warring States Period, and that they have a similar maturity with the oracle-bone inscriptions in the Yin Shang dynasty of China. Due to the similarity between the Gansang stone inscriptions and these ancient characters, we can presume that the Gansang stone inscriptions may be the writing system created and used by the ancient Luoyue ethnic group, but the scientific nature of this conclusion cannot be fully confirmed.

\subsubsection{Views of Chao Ban \& Rongqin Xiao (Researchers at Jinan University)}

Chao Ban \& Rongqin Xiao (2013) compared the Gansang stone inscriptions with ancient writing systems found in China, such as the oracle-bone inscriptions, the Naxi writing system, the Shuishu writing system, and the ancient Yi writing system. They drew the conclusion that the Gansang stone inscriptions are related to these ancient writing systems found in China. Ban and Xiao's (2013) judgment is based on the many similar symbols between the Gansang stone inscriptions and these ancient writing systems. As there are many similar symbols in the Gansang stone inscriptions and the Shuishu writing system and the age of the Gansang stone inscriptions is more ancient than the Shuishu writing system, Chao Ban \& Rongqin Xiao (2013) determined that the Gansang stone inscriptions may be an ideographic writing system used by ancient Tai-kadai ethnic groups.

The aforementioned assumptions of Ban and Xiao are based on the fact that the Gansang stone inscriptions have many similar characters with the oracle-bone inscriptions, the Shuishu writing system, and the ancient Yi writing system. However, it is not possible to determine whether these similarities are accidental or inevitable. Thus, it is only possible to conclude that there may be a connection between these writing systems. 


\subsubsection{Views of Jingfang Li \& Ye Liu (Researchers at the Minzu University of China)}

Jingfang Li \& Ye Liu (2014) compared the Gansang stone inscriptions with many ancient writing systems, pointing out that the Gansang stone inscriptions have some similar symbols as those found in the oracle-bone inscriptions, the Shuishu writing system, the ancient Yi writing system, and Harappa writing system. In addition, Jingfang Li \& Ye Liu (2014) also determined that the Gansang stone inscriptions may be a mature ideographic writing system used by the ancient Tai-kadai ethnic groups according to the characteristics of the Gansang stone inscriptions and the ancient ethnic groups' distribution in the Pingguo county area. However, it is unclear whether the similarities between these ancient writing systems is coincidental. In addition, although there is a relationship between the distribution of ethnic groups and the distribution of writing systems, the significance of the connection between them is unclear.

\subsection{A Comparative Study of the Gansang Stone Inscriptions and Other Related Ancient Writing Systems}

\subsubsection{A Comparative Study of the Gansang Stone Inscriptions and Other Ancient Luoyue Characters in Guangxi}

At present, in addition to a large number of Gansang stone inscriptions, many sporadic ancient Luoyue characters have also been found in the area in which the ancient Luoyue ethnic group is distributed. These ancient Luoyue characters are found in the Minjiang, Zuojiang, Xijiang, and Youjiang river basins and Daming mountain and Tianjing mountain in the Gaozhou and Wuming areas. According to previous research, these ancient Luoyue characters are records of the worship of the rain ( $\mathrm{Li}, 2018$, p. 9). In addition to the stone tablets, the oracle bone pieces, pottery pieces, and other various types of bone pieces are the carriers of these ancient characters. The structure of these ancient Luoyue characters is very similar to those in the Gansang stone inscriptions. Although it is impossible to confirm the connection between them, this can at least prove that the Gansang stone inscriptions found in the ancient Luoyue ethnic group distribution area is not an isolated phenomenon. This also indicates that the Gansang stone inscriptions are not relics brought by other ethnic groups (Li, 2018, p. 9). Zhiqiang Li (2018) judged that the Gansang site may have been the large-scale ritual centre of the ancient Luoyue ethnic groups, as the other ancient Luoyue characters distributed in the area are sacrificial ruins or local shrine temple ruins (p. 9).

2.5.2 A Comparative Study of the Gansang Stone Inscriptions, Oracle-Bone Inscriptions, Shuishu Writing System, and Ancient Yi Writing System

In order to interpret the Gansang stone inscriptions as quickly as possible, scholars turned their attention to other ancient writing systems found in China, hoping to gradually interpret the Gansang stone inscriptions by comparing them with these other ancient writing systems. Cao Ban (2013), Fang Qin (2013), Jinfang Li and Ye Liu (2014), Xuan Ban (2014), Zhiqiang Li (2018), and other scholars have compared the Gansang stone inscriptions with oracle-bone inscriptions, the Shuishu writing system, and the ancient Yi writing system. Scholars have found that the Gansang stone inscriptions have many similarities with the oracle-bone inscriptions; the characters in both inscriptions are all square, and there are many shared characters.

The similarity between characters in the Gansang stone inscriptions and the oracle-bone inscriptions makes it easy to draw the conclusion that the two writing systems have a homologous relationship. However, after further analysis, scholars found that the number of similar characters in the two inscriptions is relatively small, that these characters typically refer to natural phenomena or basic objects that are common in human life, and the strokes of these characters are relatively simple. Hence, this does not rule out the possibility of accidental similarity (Ban, 2013; Li \& Liu, 2014). In addition, common characters (or radicals) in the oracle-bone inscriptions, such as “人 people," “木 wood," “水 water," and “手 hands," do not appear in the Gansang stone inscriptions, which implying that the Gansang stone inscriptions and the oracle-bone inscriptions have different sources (Ban, 2013; Ban, 2014).

According to Zhiqiang Li's statistical analysis of 16,173 Gansang stone inscriptions characters included in his 2018 book of "The image narrative of Pingguo Gansang stone inscriptions: Characters copy set," only 97 characters have been found that are the same as or similar to those in the oracle-bone inscriptions; the ratio is less than $0.6 \%$ (pp. 10-13). All of the above evidence indicates that there is no homology between the Gansang stone inscriptions and the oracle-bone inscriptions. The structural features of the Gansang stone inscriptions and the results of radiocarbon dating the stone tablets indicate that the Gansang stone inscriptions present a more mature writing system that appeared in the era same as the oracle-bone inscriptions, and that there is no homologous relationship between the Gansang stone inscriptions and oracle-bone inscriptions.

In the comparison of the Gansang stone inscriptions and Shui Shu characters, scholars have also identified a certain number of characters that are the same or similar in these two writing systems. 
Characters in the Gansang stone inscriptions that share similarities with Shui Shu characters are mainly concentrated in "Heavenly Stems and Earthly Branches" words, time words, and number words (Ban, 2013; Li \& Liu, 2014). Considering these meanings, Chao Ban (2013) believes that this reflects the homologous relationship between the Gansang stone inscriptions and the Shui Shu writing system (pp. 18-20). Thus, Ban (2013) has argued that the Gansang stone inscriptions present an ancient writing system of the ancient Tai-Kadai ethnic groups (p. 21). This inference is still not fully affirmed, and further research is needed.

Scholars have found that there are fewer same or similar characters in the Gansang stone inscriptions and the ancient Yi writing system. According to Jinfang Li and Ye Liu (2014), a total of 27 same or similar characters were found in both the Gansang stone inscriptions and the ancient Yi writing system.

The strokes of the same or similar characters in both the Gansang stone inscriptions and the ancient Yi writing system are relatively simple, so the possibility of accidental similarity cannot be ruled out (Ban, 2013; Li \& Liu, 2014). In addition, no historical records of communication between the ancient Luoyue ethnic group and the ancient Yi ethnic group have been found. This indicates that, the likelihood of homology between the Gansang stone inscriptions and the ancient Yi writing system is very small.

\subsubsection{A Comparative Study of the Gansang Stone Inscriptions and the Harappa Writing System in India}

Scholars have not only compared Gansang stone inscriptions with ancient characters in China; it has also been compared with ancient characters in other countries.

Mayank Vahia (2014) and Li Jinfang and Liu Ye (2014) compared the Gansang stone inscriptions with the Harappa writing system in Indian and identified many similarities. Mayank Vahia (2014) pointed out that about $10 \%$ of the characters in the Gansang stone inscriptions are similar to Harappa characters.

Further research is needed to determine whether the similar characters that appear in the two writing systems is a kind of contingency or whether there is a relationship between the two systems.

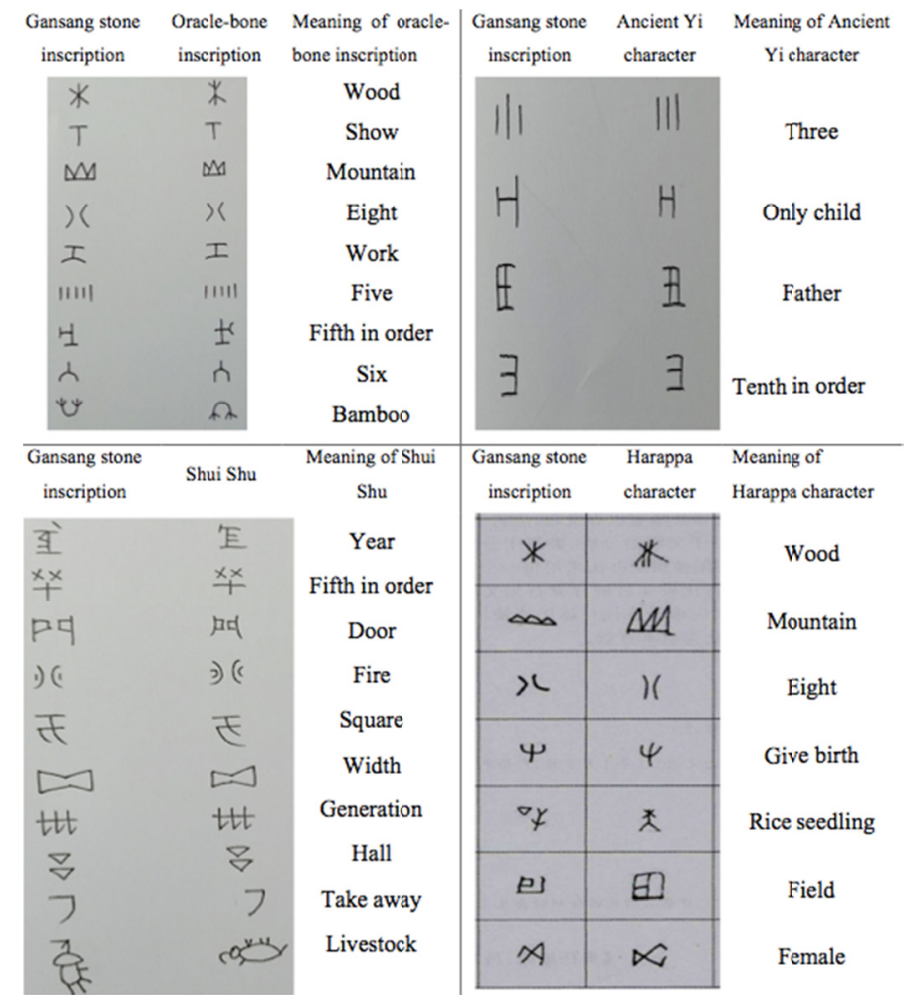

Figure 2. Sample of similar characters in the Gansang stone inscriptions and other ancient writing systems

\subsection{Gansang Stone Inscriptions: An Ancient Writing System of the Ancient Tai-Kadai Ethnic Groups?}

The consensus of scholars is that the Gansang stone inscriptions present an ancient writing system of the ancient Tai-Kadai ethnic groups in almost the same era of the oracle-bone inscriptions. As a writing system with the same maturity as that of the oracle-bone inscriptions, the Gansang stone inscriptions contradict previous 
assumptions that the ancient Tai-Kadai ethnic groups had no writing system (Ban, 2013; Qin, 2013; Li \& Liu, 2014; Li, 2016, 2018). In addition, on October 31, 2018, Jinfang Li gave a special academic report at Yunnan Normal University: "From the Gansang stone inscriptions to the Ninglang ancient character: The self-originating writing systems chain of ancient Tai-Kadai ethnic groups?" In this report, Li pointed out that by comparing the Gansang stone inscriptions with ancient writing systems (or characters) found in the Lingnan area of China, it can be speculated that the ancient Tai-Kadai ethnic groups created their own national writing systems (or characters). Li stated that these ancient writing systems (or characters) found in the Lingnan area may be the writing systems (or characters) created by the ancient Tai -Kadai ethnic groups in different periods as well as in different regions, and they are very likely to be self-originated writing systems (or characters).

Of course, the evidence that supports the assertion that the Gansang stone inscriptions are the writing system of ancient Tai-Kadai ethnic groups is slightly weak. While the location in which the Gansang stone inscriptions were discovered was the dwelling place where the ancient Tai-Kadai ethnic groups (Luoyue ethnic group) for generations, and the Gansang stone inscriptions has a certain degree of similarity with some ancient writing systems associated with the ancient Tai-Kadai ethnic groups, scholars have failed to find more evidence to prove that the Gansang stone inscriptions are the writing system of the ancient Tai-Kadai ethnic groups. The best way to answer this question is to interpret the Gansang stone inscriptions correctly; this will allow us to accurately judge the nature of the Gansang stone inscriptions through their content. Currently, due to limited data, it appears that correctly interpreting the Gansang stone inscriptions will be a long process.

In addition, scholars are also considering whether the Gansang stone inscriptions are a phonography or ideogram. Through analysis of the Gansang stone inscriptions, and drawing on research on the oracle-bone inscriptions, scholars have pointed out that Gansang stone inscriptions should be ideograms (Ban, 2013; Li \& Liu, 2014). Chao Ban (2013) suggested that the Gansang stone inscriptions have a self-explanatory character and associate compounds character, but no pictophonetic characters (p. 21). Jinfang Li and Ye Liu (2014) divided the Gansang stone inscriptions into two types, including the single character and the combined character, and further divided the combined characters into 18 types by different combinations of upper, lower, left and right, and inside and outside; they also found 28 commonly used and highly productive character components. Because some of the character components appear repeatedly in different characters, Jinfang Li and Ye Liu (2014) pointed out that the Gansang stone inscriptions present a relatively mature ideogram, similar to that of the oracle-bone inscriptions.
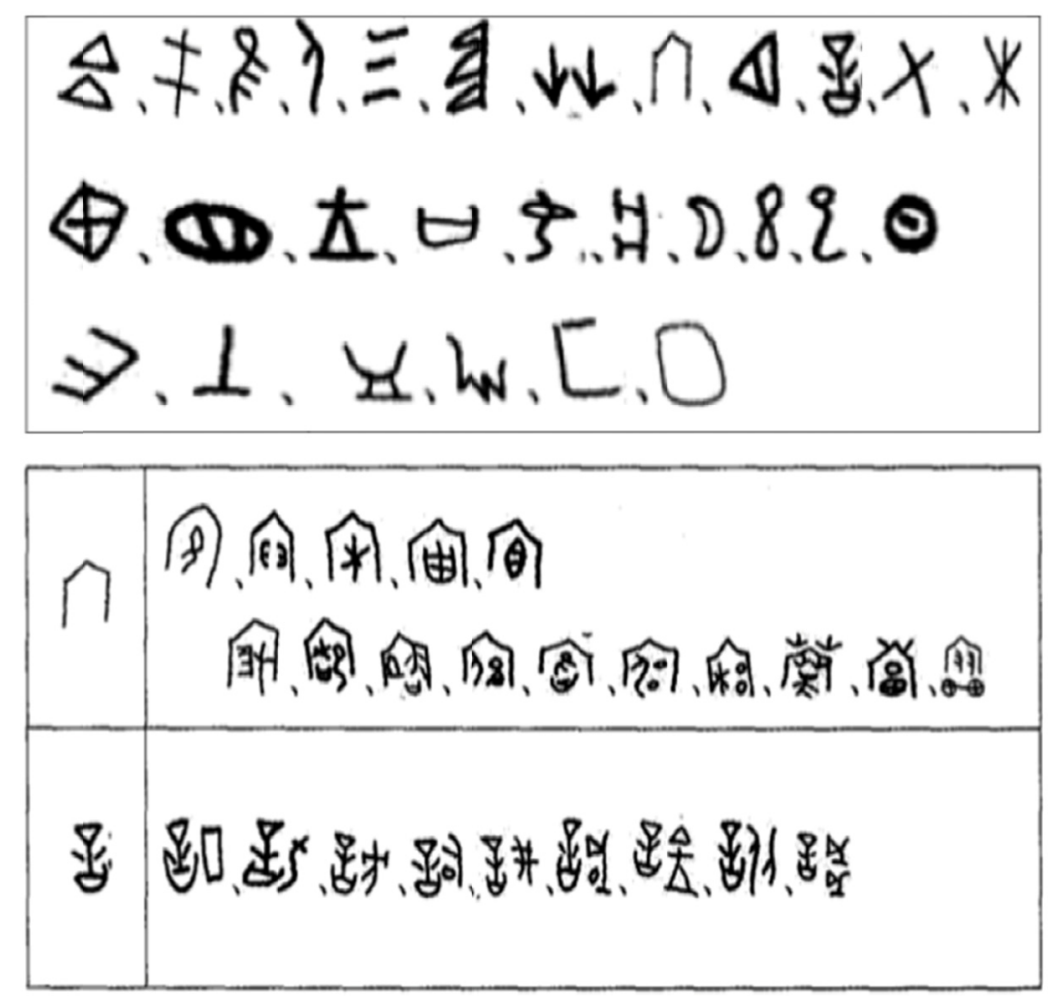

Figure 3. 28 commonly used and highly productive character components of the Gansang stone inscriptions and Examples of characters that have the same component (Li \& Liu, 2014) 
From the characteristics of the Gansang stone inscriptions we have found so far, we can indeed confirm that the inscriptions are an ideogram, and there is no characteristic of the phonography. However, further research is required to determine how the meanings are expressed by the characters and the connections between characters and meanings.

\section{Textual Reading of the Gansang Stone Inscriptions}

In textual readings of the Gansang stone inscriptions, scholars have mainly paid attention to the stroke order and the order of the lines of the characters in the inscriptions. Magnifying the font of the Gansang stone inscriptions shows that in vertical strokes, the upper part of the stroke is deeper and thicker and the lower part of the stroke is lighter and thinner. In horizontal strokes, the left part of the stroke is deeper and thicker, while the right part of the stroke is lighter and thinner. In left-falling strokes, the upper right part of the stroke is deeper and thicker and the lower left part of the stroke is lighter and thinner. In right-falling strokes, the upper left part of the stroke is deeper and thicker and the lower right part of the stroke is lighter and thinner. These characteristics indicate that the basic stroke order of the Gansang stone inscriptions involves vertical strokes from top to bottom, horizontal strokes from left to right, left-falling strokes from top right to bottom left, and right-falling strokes from top left to bottom right. However, when horizontal strokes, vertical strokes, left-falling strokes, and right-falling strokes are present in the same character, we cannot determine the order in which these strokes are written.

Scholars have a more consistent perspective regarding the order of the Gansang stone inscriptions; scholars agree that the Gansang stone inscriptions are arranged from top to bottom (Ban, 2013; Li \& Liu, 2014; Li, 2018). No scholars have raised objections to this perspective, and this assumption is consistent with the textual arrangement characteristics of the currently discovered Gansang stone inscriptions. At the same time, this assumption is also consistent with people's cognitive laws and engineering mechanics principles (Ban, 2013, p. 9).

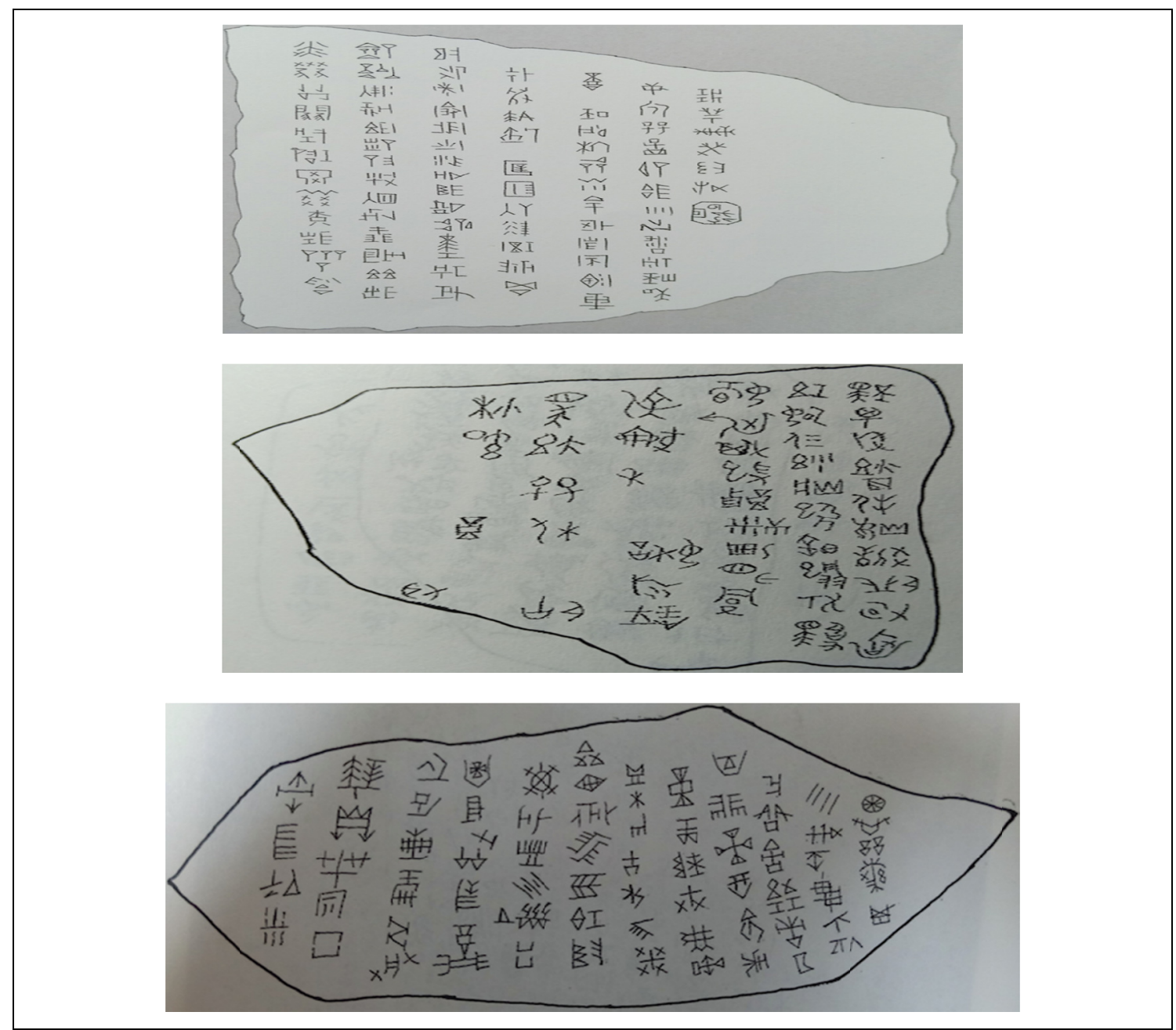

Figure 4. Examples of text arrangement of Gansang stone inscriptions (Ban, 2013, p. 9; Li, 2018, p. 33, 105) 
However, the views of scholars have not yet been unified considering whether the current texts of the Gansang stone inscriptions are arranged from left to right or right to left. Chao Ban (2013) determined that the texts of the Gansang stone inscriptions should be arranged from left to right according to the characteristics of the stone tablets that he collected, in which the right blank space is typically larger than the left blank space (p. 10). At the same time, Chao Ban (2013) also pointed out that the arrangement order of the text should be final confirmation after a certain Gansang stone inscriptions are correctly interpreted (p. 10).

Jinfang Li and Ye Liu (2014) pointed out that most people use their right hand when writing, and they are used to writing from near to far, which is convenient and labor-saving. Considering these habits that are common among human beings, it can be assumed that the arrangement of the Gansang stone inscriptions should be arranged from right to left.

In addition, Zhiqiang Li (2018) determined that the texts of the Gansang stone inscriptions should be arranged from right to left due to the characteristics of the inscriptions on the stone tablets that he collected, in which the right blank space is typically larger than the left blank space, and because the writing style of ancient Chinese characters is from right to left (p. 24).

Considering the Gansang stone inscriptions that have been acquired, it is clear that the size of the blank space on the left and right sides of the stone tablets is not sufficient as the basis for determining the orientation of the Gansang stone inscriptions. Some of the Gansang stone inscriptions that have been collected thus far have larger blank spaces on the right side, some have larger blank spaces on the left side, and some have almost the same size of blank space on the left and right sides. The human writing habits pointed out by Jinfang Li and Ye Liu (2014) and Zhiqiang Li's (2018) assertion that the writing style of ancient Chinese characters is from right to left can be used as references, but are not sufficient evidence to determine whether the arrangement of the Gansang stone inscriptions must be arranged from right to left. To determine the arrangement of the Gansang stone inscriptions, accurate judgments must be made based on the contextual meaning of the inscriptions after they have been correctly interpreted.

\section{Character and Civilization: Exploring the Tai-Kadai Ethnic Groups' Civilization from the Gansang Stone Inscriptions}

The location of the discovery of the Gansang stone inscriptions falls within the jurisdiction of the ancient Luoyue joint city-state country. This area was the location of a kingdom established by the ancient Luoyue ethnic group (a branch of the ancient Tai-Kadai ethnic groups), who have been present in the area for more than one thousand years. The ancient Luoyue ethnic group created the "Rice culture," the "Stone shovel culture," the "Dragon mother culture," the "Bronze culture," the "Bronze drum culture," the "Huashan culture," the "Mountain song culture," the "Picture song on the slopes culture," and the "Women's sun culture." Through an examination of nearly 1,000 ancient tombs from the Shang and Zhou Dynasties that were discovered in Matou town, Wuming County, Chaoxiong Zheng (2005) determined that Matou town was the capital of the ancient Luoyue joint city-state country (p. 209). A large number of writing symbols found in the Youjiang River Basin, which was under the jurisdiction of the ancient Luoyue joint city-state country, have been identified by experts as the writing system of the ancient Luoyue ethnic group. Some of these inscriptions are engraved on stone, some are engraved on pottery, some are engraved on jade, and some are engraved on bone. Although these writing systems found in the location of the ancient Luoyue joint city-state country are not quite the same as the writing in the Gansang stone inscriptions, this illustrates that the Gansang stone inscriptions in the area of the ancient Luoyue joint city-state country are not isolated. It can be presumed that the appearance of the Gansang stone inscriptions was the product of the powerful economic, political, and cultural background of the ancient Luoyue joint city-state country (Li, 2018, p. 19).

Previous studies on the ancient civilization of the Tai-Kadai ethnic groups, on the one hand, have mainly relied on Chinese literature, such as "Zhou Shu (The book of Chow)," "Han Shu • Di Li Zhi (The book of Han • Geography)," "Shi Ji • Nan Yue Lie Zhuan (Shi Ji • Biography of Nan Yue)," "Han Shu • Ma Yuan Zhuan (Han Shu • Biography of Ma Yuan)," "Yue Jue Shu (The history of Yue area)," "Jiao Zhou Wai Yue Ji (Biography of Jiao Zhou)," and "Jiu Tang Shu • Di Li Zhi (The Book of Old Tang • Geography)." On the other hand, such work must rely on archaeological excavations. Since there was very little contact between the ancient Tai-Kadai ethnic groups and ethnic groups in the Central Plains of China, Chinese literature contains very few records of the ancient Tai-Kadai ethnic groups, and many of the records of the Tai-Kadai ethnic groups in Chinese literature display ethnic prejudice. Therefore, we cannot accurately understand the civilization of the ancient Tai-Kadai ethnic groups through these records. Regarding archaeological pursuits, many sites of ancient civilizations have been discovered in the area in which the ancient Tai-Kadai ethnic groups lived. However, due to the lack of 
relevant literature, it has not been possible to gather information about the civilization of Tai-Kadai ethnic groups from many of these archaeological discoveries.

The appearance of the Gansang stone inscriptions has opened a new door for the exploration of the civilization of the ancient Tai-Kadai ethnic groups. The Gansang stone inscriptions were discovered in the former territory of the Luoyue joint city-state country; it is likely that they record the politics, economics, and culture of ancient Tai-Kadai ethnic groups. The interpretation of the Gansang stone inscriptions will help us further explore the early civilization of the ancient Tai-Kadai ethnic groups.

Zhiqiang Li (2018) made a preliminary interpretation of the Gansang stone inscriptions after comparing them with the oracle-bone inscriptions, pointing out that the Gansang stone inscriptions recorded people's apparel, architecture, residence, eating habits, transportation, hunting activities, war, raising livestock, sacrifice, divination, astronomy, and calendar (pp. 26-31).

Of course, this is only a preliminary interpretation based on the pictographic meaning of the characters present in the Gansang stone inscriptions and in reference to the results of research on the oracle-bone inscriptions. From this preliminary interpretation, it is clear that the content of the Gansang stone inscriptions may be very rich. Once the Gansang stone inscriptions have been completely interpreted, they will present us with a vivid image of an ancient civilization, pushing our exploration of the Tai-Kadai ethnic groups to a new height and making it possible to rewrite the civilization history of the Tai-Kadai ethnic groups. In turn, it will provide us with a broader perspective and valuable literature for the study of the origins of human civilization.

\section{Conclusion and Discussion}

The discovery of the Gansang stone inscriptions is one of the most important discoveries of the 21st century; it has opened a new door of hope for the exploration of ancient human civilization. Although scholars have not yet been able to fully interpret the Gansang stone inscriptions due to the lack of literature material, some preliminary results have been achieved. Considering the distribution of ancient ethnic groups in the discovery location of the Gansang stone inscriptions, the age of the stone tablets, and the similarities between the Gansang stone inscriptions and other confirmed writing systems of ancient Tai-Kadai ethnic groups, scholars have preliminarily identified the Gansang stone inscriptions as a kind of writing system used by the ancient Tai-Kadai ethnic groups.

From the number of characters that have been collected from the Gansang stone inscriptions and the characteristics of these characters, we can judge that this is a very mature ideographic writing system. At present, scholars can only interpret some individual characters from the Gansang stone inscriptions according to their pictographic meanings as well as by referring to oracle-bone inscriptions research results, and cannot interpret any full texts.

We believe that the interpretation of the Gansang stone inscriptions cannot be accomplished by linguists alone; linguists, ethnologists, and historians must work together because it is only possible to fully interpret the Gansang stone inscriptions through comprehensive interdisciplinary research. Although the interpretation of the Gansang stone inscriptions will require a long process involving considerable manpower and economic input, for the exploration of human civilization, any effort is worthwhile.

\section{References}

Ban, C. (2013). The copy sheet and character set of Gansang stone inscriptions. Guangzhou: Guangdong Science and Technology Press. (In Chinese)

Ban, C., \& Xiao, R. Q. (2013). Preliminary study on the carving of Gansang stone inscriptions. Cultural heritage, 13(5), 100-105. (In Chinese). https://doi:10.3969/j.issn.1674-0890.2013.05.014

Ban, X. (2014). A comparative study of Gansang stone inscriptions and oracle-bone inscriptions. Language and Literature, 17, 38-40. (In Chinese). https://doi:10.3969 /j.issn.1672- 8610. 2014.17.015

Dai, Q. X. (2013). Preface for "The copy sheet and character set of Gansang stone inscriptions." In C. Ban, The copy sheet and character set of Gansang stone inscriptions (pp. 1-2). Guangzhou: Guangdong Science and Technology Press. (In Chinese)

He, Y. D. The relationship between marine culture of Austronesian language family and Luoyue ethnic group. In B. Z. Jiang (Ed), Baiyue culture research (pp. 135-145). Xiamen: Xiamen University Press. (In Chinese)

Huang, M. B. (2006). The photocopy of Buluotuo scriptures. Nanning: Guangxi People's Publishing House. (In Chinese) 
Jiang, T. Y. The study of Gansang stone inscriptions. In Proceedings of Zhuang ethnic folk ancient scripts and the Gansang stone inscriptions research work symposium, Baise, China, 25-27 January 2013 (pp. 57-59). Baise: Baise University. (In Chinese)

Li, J. F., \& Liu, Y. (2014). An initial analysis on Gansang stone inscriptions. Journal of Baise University, 27(4), 45-54. (In Chinese). https://doi:10.3969/j.issn.1673- 8233.2014.04.009

Li, J. F. (2018). From the Gansang stone inscriptions to the Ninglang ancient characters: The self-originating writing systems chain of ancient Tai-Kadai ethnic groups? Academic report at Yunnan Normal University, Kunming, China, 31 October 2018. (In Chinese)

Li, Z. Q. (2016). The image narrative and cultural tradition of Pingguo Gansang stone inscriptions. National literature research, 34(2), 49-55. (In Chinese). https: // doi: 10.3969/j.issn.1002- 9559.2016.02.005

Li, Z. Q. (2018). The image narrative of Pingguo Gansang stone inscriptions: Characters copy set. Beijing: China Social Sciences Press. (In Chinese)

Liang, T. W. (2013). Gansang cared symbols: The characters of ancient Luoyue is immortal. In Proceedings of Zhuang ethnic folk ancient scripts and the Gansang stone inscriptions research work symposium, Baise, China, 25-27 January 2013 (pp. 1-3). Baise: Baise University. (In Chinese)

Liang, T. W. (2018). Preface for "The image narrative of Pingguo Gansang stone inscriptions: Characters copy set." In Z. Q. Li, The image narrative of Pingguo Gansang stone inscriptions: Characters copy set (pp.1-5). Beijing: China Social Sciences Press. (In Chinese)

Literatures of Gansang stone inscriptions. Collected by Propaganda Department of Pingguo county, 25 January 2013. (In Chinese)

Qin, F. (2013). Interpretation of Gansang stone inscriptions. In Proceedings of Zhuang ethnic folk ancient scripts and the Gansang stone inscriptions research work symposium, Baise, China, 25-27 January 2013 (pp. 36-38). Baise: Baise University. (In Chinese)

Qin, Z. Q. (2013). The report of the identification of Gansang stone inscriptions by Yi (Lolo) expert. In Proceedings of Zhuang ethnic folk ancient scripts and the Gansang stone inscriptions research work symposium, Baise, China, 25-27 January 2013 (pp. 13-15). Baise: Baise University. (In Chinese)

Vahia, M. (2014). Written material found at Gansang. In Proceedings of international conference for Gansang stone inscriptions, Pingguo, China, 24-26 June 2013 (pp. 21-23). Pingguo: Pingguo County Government.

Zheng, C. X. (2005). The origin and development conditions of Liao songs of Zhuang ethnic group. Guangxi Ethnic Studies, 5(1), 94-102. (In Chinese). https: // doi: 10.3969/j.issn.1004-454X. 2005.01.014

Zheng, C. X. (2005). Research on the origin of Zhuang ethnic group's civilization. Nanning: Guangxi People's Publishing House. (In Chinese)

Zheng, C. X. (2014). The study of Gansang stone inscriptions found in Pingguo county. In Proceedings of international conference for Gansang stone inscriptions, Pingguo, China, 24-26 June 2013 (pp. 71-73). Pingguo: Pingguo County Government. (In Chinese)

\section{Copyrights}

Copyright for this article is retained by the author(s), with first publication rights granted to the journal.

This is an open-access article distributed under the terms and conditions of the Creative Commons Attribution license (http://creativecommons.org/licenses/by/4.0/). 